Review

\title{
Pharmacological Actions of Multi-Target-Directed Evodiamine
}

\author{
Hui Yu ${ }^{1,2}$, Hongwei Jin ${ }^{3}$, Wuzhuang Gong ${ }^{4}$, Zhanli Wang ${ }^{4, *}$ and Huaping Liang ${ }^{1, *}$
}

1 State Key Laboratory of Trauma, Burns and Combined Injury, Research Institute of Surgery,

Daping Hospital, the Third Military Medical University, Chongqing 400042, China;

E-Mail: huiyu2008@hotmail.com

2 The Second Affiliated Hospital, Baotou Medical College, Baotou 014030, China

3 State Key Laboratory of Natural and Biomimetic Drugs, Peking University Health Science Center, Beijing 100191, China; E-Mails: jinhw@bjmu.edu.cn

4 The First Affiliated Hospital, Baotou Medical College, Baotou 014010, China; E-Mail: gongwz73@sina.com

* Authors to whom correspondence should be addressed; E-Mails: wang.zhanli@hotmail.com (Z.W.); huaping_liang@yahoo.com.cn (H.L.); Tel.: +86-0472-217-8195 (Z.W.);

Fax: +86-0472-212-9235 (Z.W.); Tel.: +86-023-6875-7411 (H.L.); Fax: +86-023-6875-7404 (H.L.).

Received: 14 December 2012; in revised form: 28 January 2013 / Accepted: 29 January 2013 /

Published: 31 January 2013

\begin{abstract}
Evodiamine, a naturally occurring indole alkaloid, is one of the main bioactive ingredients of Evodiae fructus. With respect to the pharmacological actions of evodiamine, more attention has been paid to beneficial effects in insults involving cancer, obesity, nociception, inflammation, cardiovascular diseases, Alzheimer's disease, infectious diseases and themoregulative effects. Evodiamine has evolved a superior ability to bind various proteins, so we also argue that it is good starting point for multi-target drugs. This review is primarily addressed to the description of the recent advances in the biological activity studies of evodiamine, with a focus on pharmacological mechanism. The present review also includes the pharmacokinetics and the detailed exploration of target-binding properties of evodiamine in an attempt to provide a direction for further multi-target drug design.
\end{abstract}

Keywords: evodiamine; bioactivity; mechanism; receptor binding 


\section{Introduction}

Many common diseases like diabetes, cardiovascular disease, and cancer are caused by a set of several factors, such as physiological, pathological, environmental, and lifestyle. In the past, the main effort was aimed at developing highly specific molecules acting on single targets [1]. Now, there is a general agreement that molecules interfering simultaneously with multiple targets might be more effective than single target agents [2]. Moreover, using such a multi-targeted approach could theoretically permit low-dose administration of active agents therefore reducing the potential undesired events by providing synergistic or additive preventive effects. Chinese herbal medicines are attracting intensive attention because of their history of reliable therapeutic efficacy for the prevention and treatment of various human diseases for thousands of years [3-6]. Phytochemicals from medicinal plants play a vital role in treating diseases by influencing the function of a number of diverse targets. Therefore, Chinese herbal medicines are becoming important resources for designing multi-target bioactive molecules. Accordingly, it is worth noting that genomic, proteomic, and computational approaches have been explored to identify the molecular targets of natural products. Over the past decade, more and more molecular targets of certain natural herbal product have been proposed, which is helpful for current multi-target drug discovery [7]. For example, the Zhang research group investigated the therapeutic mechanisms of astragaloside IV extracted from Astragalus membranaceus Bunge, a medicinal herb used for cardiovascular diseases. They identified a total of 39 putative targets of astragaloside IV, implicating that the therapeutic effects of astragaloside IV are based upon a combination of blocking calcium influx, vasodilation, anti-thrombosis, anti-oxidation, anti-inflammation and immune regulation [8].

The fruit of "Wu-Zhu-Yu" (Evodiae fructus; Evodia rutaecarpa Benth., Rutaceae) is one of the most popular and multi-purpose herbs traditionally used in China for the treatment of headaches, abdominal pain, difficult menstruation, vomiting, diarrhea, and other diseases [9]. Phytochemical studies have shown the presence of evodiamine (Figure 1), which is an indole alkaloid found in large amounts in the Chinese medicine evodia [10].

Figure 1. Structure of evodiamine.

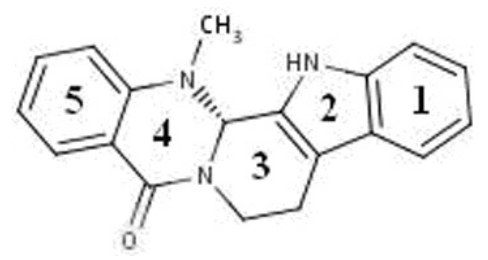

Growing evidence demonstrates that evodiamine represents an important compound possessing a wide spectrum of biological activities [11-14], suggesting that it might interact with a number of diverse targets to carry out its therapeutic effects. The broad spectrum of medicinal properties associated with this compound has encouraged medicinal chemists to design and synthesize a large number of novel therapeutic agents. Several analogues exhibit significant anti-tumour, anti-microbial, and anti-inflammatory activities. For this reason evodiamine is an object of continuously growing interest amongst the scientists. With the characterization of the molecular targets for evodiamine, evodiamine can be used as a promising scaffold for development of a novel class of multi-target-directed 
compounds, which can be beneficial for cancer or inflammatory treatment. This review attempts to summarize the recent researches on evodiamine focusing on biological activity and mechanism of action. Moreover, target-binding properties and bioavailability of evodiamine were also highlighted.

\section{Biological Activities of Evodiamine}

\subsection{Anti-Inflammatory Activity}

An appreciable amount of research has reported on the potential anti-inflammatory properties and the possible underlying mechanisms of action of evodiamine. Nitric oxide (NO) is a highly reactive molecule produced from the amino acid arginine by the enzyme NO synthase (NOS). Inappropriate, excessive production of NO is largely responsible for pathogenesis of various inflammatory diseases [15]. Chiou et al., examined the possible anti-inflammatory effects of evodiamine by assessing its effects on NO production in cultured murine macrophage-like cell line RAW 264.7 [16]. Their results indicated that evodiamine inhibited NO production by interfering with the interferon-gamma (IFN- $\gamma$ )-initiated signaling events. Ko et al., also found that anti-inflammatory activities of evodiamine could be partially explained by its potentials for inhibiting inducible nitric oxide synthase (iNOS)-dependent NO production in activated inflammatory cells [17]. In addition, evodiamine was found to inhibit the action of nuclear factor kappa $\mathrm{B}(\mathrm{NF}-\mathrm{\kappa B})$ and the transcription of cyclo-oxygenase-2 (COX-2). Liu et al. recently demonstrated that evodiamine inhibited $\mathrm{COX}-2$ expression and hypoxia-inducible factor $1 \alpha$ (HIF-1 $\alpha$ ) accumulation via dephosphorylation of the serine/threonine protein kinase B (PKB/Akt) and the $70 \mathrm{kDa}$ ribosomal S6 kinase (p70S6k), providing evidence for a novel mechanism underlying its anti-inflammatory activity [18]. Moreover, evodiamine has strong inhibitory effects on the synthesis of prostaglandin $\mathrm{E}_{2}\left(\mathrm{PGE}_{2}\right)$, which is the principal pro-inflammatory prostanoid and contributes to one of the key features of inflammation [19]. These results provide a scientific rationale for the anti-inflammatory use of evodiamine. The central role of evodiamine in the regulation of inflammatory is summarized in Figure 2.

Figure 2. Demonstration of the key role of evodiamine in the regulation of inflammatory. $\mathrm{T}$-lines indicate inhibitory effects.

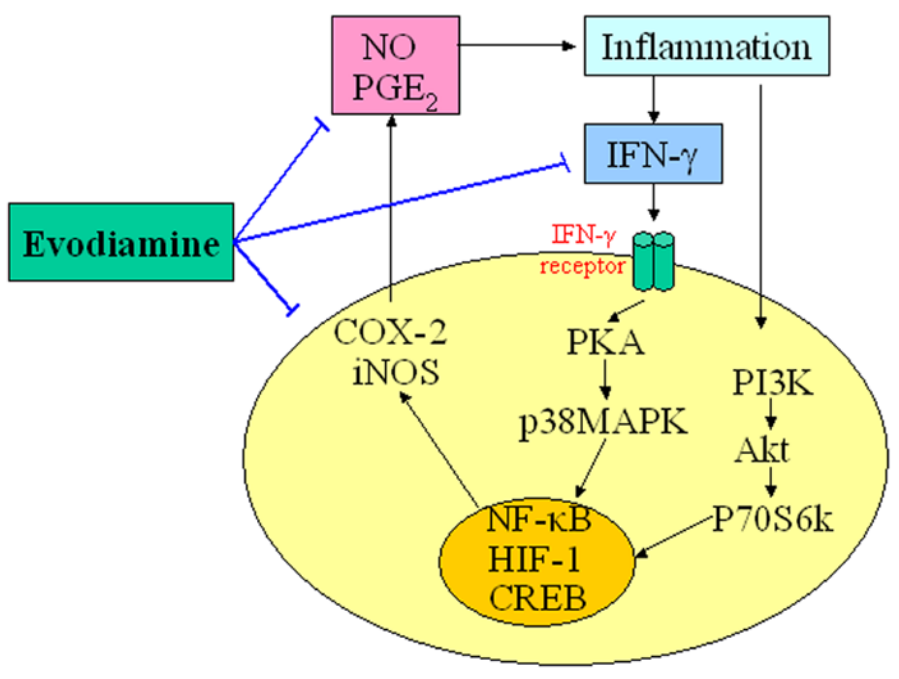




\subsection{Anti-Cancer Activity}

Evodiamine has been shown to exhibit anti-tumor properties by inhibiting proliferation of various cancer cell lines, including cervical cancer cells, colon cancer cells, lung cancer cells, melanoma cells, leukemic T-lymphocyte cells, prostate cancer cells and breast cancer cells [20-28]. One well-known manner of suppressing proliferation rates by evodiamine involves cell cycle progression arrest $(\mathrm{G} 2 / \mathrm{M}$ phase) via activation of $\mathrm{Cdc} 2 /$ cyclin B [29]. In addition, evodiamine induced apoptosis of a variety of tumor cell lines through several pathways [30-32]. Takada et al. found that evodiamine exhibited

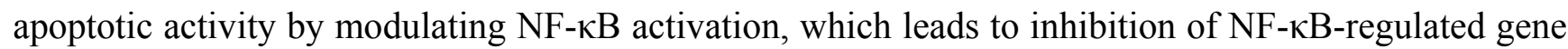
products such as Cyclin D1, X chromosome-linked IAP (XIAP), Bcl-2, and Bcl-X1 [33]. Other studies further revealed that evodiamine increased the expression of the apoptosis inducer Bax and decreased that of the apoptosis suppressor Bcl-2, and then induced apoptosis through the caspase pathway $[34,35]$. Reactive oxygen species (ROS) and NO generations were also found to exhibit regulatory effects on functions of p53, p21, protein tyrosine kinase (PTK) and other signaling proteins involved in evodiamine-induced apoptosis [36-39]. PI3K/Akt and extracellular signal-regulated kinases (ERKs) signaling pathways were also found to exhibit essential roles in the responses of tumor cells apoptosis induced by evodiamine [40-43]. Besides its anti-proliferative and anti-apoptotic effects, the effective inhibition of tumor invasion and metastasis are additional mechanisms by which evodiamine may communicate to halt the cancerous process [44]. Moreover, evodiamine has recently been identified as a dual catalytic inhibitor of topoisomerases I and II [45-48]. Figure 3 showed the potential anti-tumor properties and the possible underlying mechanisms of evodiamine.

Figure 3. Demonstration of the potential anti-tumor properties and the possible underlying mechanisms of evodiamine. T-lines indicate inhibitory effects.

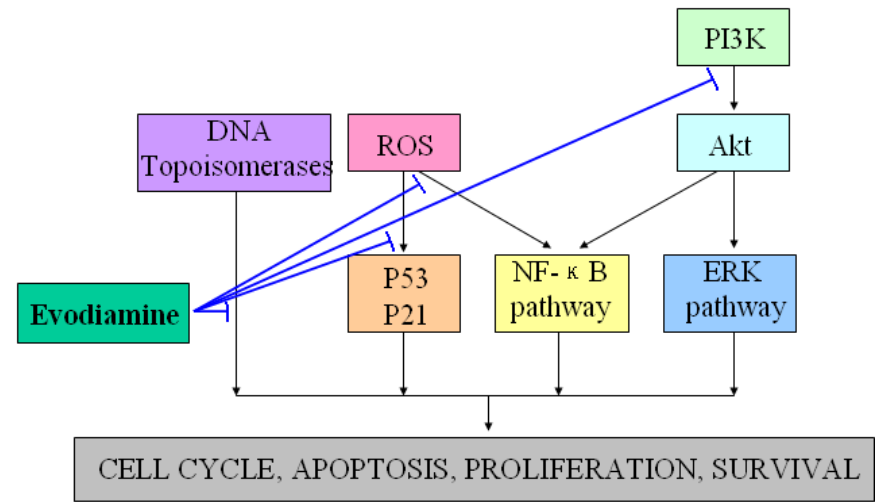

\subsection{Anti-Obesity Activity}

Evodiamine has excellent potential as an agent to prevent obesity [49]. Kobayashi et al. found that evodiamine appeared to prevent obesity and reduce body fat [12]. The major mechanism eliciting the effect was postulated to be enhancement of uncoupling protein-1 (UCP1) thermogenesis through $\beta 3$-adrenergic stimulation in brown adipose tissue (BAT). Shi et al. reported that intragastric administration of evodiamine suppressed the neuropeptide Y (NPY) mRNA and peptide levels in the arcuate nucleus (ARC) of the hypothalamus, which might be one of the mechanisms by which evodiamine exerted its fat loss effects [50]. In addition, Wang et al. reported that evodiamine inhibited adipogenesis by 
simulating the ERK/MAPK signaling pathway, which modulated the expression of the adipocyte specific transcription factors and Akt signaling [51]. They also found that evodiamine improved leptin resistance and insulin sensitivity in the mice [52]. Moreover, evodiamine inhibited both gastric emptying and gastrointestinal transit, whereas increased the plasma concentration of cholecystokinin (CCK) in a dose-dependent manner, which plays a key role in regulation of digestion and appetite $[53,54]$.

\subsection{Anti-Cardiovascular Disease Activity}

Evodiamine showed a beneficial effect on cardiovascular diseases as reported previously [55-57]. Chiou et al., found that evodiamine had a vasodilatory effect in rat isolated mesenteric arteries and the effect was endothelium dependent [58]. Evodiamine also demonstrated significant diuretic effect due to the inhibition of aldosterone release, which can control blood volume [59,60]. In addition, several studies showed that evodiamine produced transient positive inotropic and chronotropic effects on the guinea-pig isolated atria [61]. Previous investigations also indicated that evodiamine possessed a protective effect of cardiac anaphylactic injury by stimulation of calcitonin gene-related peptide (CGRP) release [62-64]. Similarly, evodiamine exerted protection against myocardial ischemia-reperfusion injury in rats by activation of vanilloid receptors to stimulate the CGRP release $[65,66]$. Moreover, evodiamine inhibited LIGHT-induced production of chemokine receptor (CCR) 1, CCR2, intracellular adhesion molecule 1 (ICAM-1), the phosphorylation of ERKs and p38 MAPK via decreasing ROS production and NADPH oxidase activation, implicating that evodiamine has the potential for use as anti-atherosclerosis agents [67].

\subsection{Anti-Alzheimer's Disease Activity}

Alzheimer's disease (AD) is a progressive, irreversible brain disease caused by degeneration of synapses and death of neurons, resulting in cognitive, memory and behavioral impairments [68,69]. It is reported that a variety of biologically active constituents isolated from traditional Chinese herbs can significantly reduce the risk of AD [70-75]. Recently, the effect and the possible mechanisms of action of evodiamine in $\mathrm{AD}$ mouse models were investigated [76]. Evodiamine was reported to improve the cognitive abilities in the transgenic models of AD. Further investigations demonstrated that evodiamine treatment increased glucose uptake in brain tissue. The results also indicated that evodiamine inhibited the expression of COX-2 and inflammatory cytokines in AD mouse models, such as IL-1 $\beta$, IL-6, and TNF- $\alpha$. However, evodiamine had no effect on amyloid beta-peptide (A $\beta$ ) deposition [76]. Therefore, the effect of evodiamine on improvement of behavior in AD mouse models was likely mediated through the inhibition of the inflammatory process. Moreover, previous investigations indicated that dehydroevodiamine, an evodiamine analogue also demonstrated significant effect on AD. The chemical structure of dehydroevodiamine has been used as a basis in the clinical development of novel cholinesterase inhibitors [77].

\subsection{Anti-Microbial Activity}

Anti-microbial agents for the treatment of infections caused by bacteria, fungi and protozoa are different from the pharmacodynamic agents that affected the physiological, biochemical, or 
immunological function of host. The increase in number of antibiotic-resistant pathogenic bacteria has stimulated research on the development and application of new antimicrobial agents. Some compounds isolated from Evodia rutaecarpa were found to act as new anti-infectious agents, such as 3-dimethylallyl-4-methoxy-2-quinolone, 1-methyl-2-pentadecyl-4(1H)-quinolone, evocarpine, dihydroevocarpine and 1-methyl-2-[(Z)-8-tridecenyl]-4-(1H)-quinolone [78-82]. Recently, Chiou et al. found that the compound evodiamine was effective in suppressing H1N1-induced chemokines production and blocking chemokine-attracted leukocytes recruitment, implicating potential in influenza virus infection-related inflammatory disorders [83]. Our group also found that evodiamine was capable of deactivation of $E$. coli and can be used as a natural antimicrobial agent. These results have not been reported yet.

\subsection{Other Activities}

It is well known that transient receptor potential cation channel subfamily V member 1 (TRPV1) plays a fundamental role in modulation of pain [84]. Some studies demonstrated that evodiamine exerted the analgesic effects due to its vanilloid receptor agonistic activities [85-87]. Evodiamine possesses many other biological functions, such as thermoregulatory effect, antianoxic action, dermatological applications, bronchoconstrictive action, and hormones secretion [88-96].

\section{Protein-Ligand Interaction}

Identification of molecular targets of evodiamine is an enormous opportunity for modern pharmacology. Up to data, three proteins are believed to be direct targets of evodiamine, including TRPV1, the aryl hydrocarbon receptor (AhR), and topoisomerases I and II. These proteins seem to be important in inflammation, cancer and other diseases. In fact, compounds which can regulate multiple targets may have superior utility over single-target drugs. For example, Guerrant et al. synthesized dual-acting histone deacetylase and topoisomerase II inhibitors, which potently inhibit the proliferation of representative cancer cell lines [97]. Recently, the structures of several complexes of evodiamine with its targets have been elucidated. Investigations of the interaction between evodiamine and various targets provided detailed information regarding the structural features required for binding, which can be exploited in future multi-target drug discovery strategies.

\subsection{TRPVI}

TRPV1, a member of the transient receptor potential superfamily, is activated by protons, heat, endogenous substances and natural ligands such as capsaicin, resiniferatoxin, and evodiamine. The receptor participated in a wide variety of pathological and physiological processes, suggesting that regulation of this receptor activation should have considerable therapeutic utility. TRPV1 has been linked to processes mediating inflammation, cancer, cardiovascular diseases, obesity, skin diseases, and neuropathic pain [98-106]. TRPV1 ligands have attracted much attention as promising drug candidates to block related pathological states associated with this receptor. Evodiamine is a vanilloid receptor agonist, and thus represents a new potential class of lead molecules for new analgesics development [86]. Recently, our group investigated the precise interaction between evodiamine and 
TRPV1 using homology modeling, molecular docking, dynamics simulation and pharmacophore modeling methods [107]. Our results showed that the ring 1 of evodiamine pointed toward Tyr511, establishing a hydrophobic interaction. The ring 5 of evodiamine pointed toward Tyr555, forming aromatic $\pi-\pi$ interactions. In addition, evodiamine made two H-bonds between carbonyl oxygen and amino group of Lys571 and between indol nitrogen and backbone of Ile569. Pharmacophore modeling provided further evidence for the validity of the docking studies. This study identified the structural determinants required for the interaction between TRPV1 and evodiamine, and gave new suggestions for the rational design of novel TRPV1 ligands. The biological activity experiment showed that evodiamine bound to rat TRPV1 with a $\mathrm{Ki}$ of $5.95 \pm 0.87$ microM, and yielded an $\mathrm{EC}_{50}$ value of $856 \pm 43 \mathrm{nM}$ [85]. Figure 4 showed the predicted binding mode of evodiamine in the active site of vanilloid receptor TRPV1.

Figure 4. Binding mode of evodiamine in the active site of vanilloid receptor TRPV1.

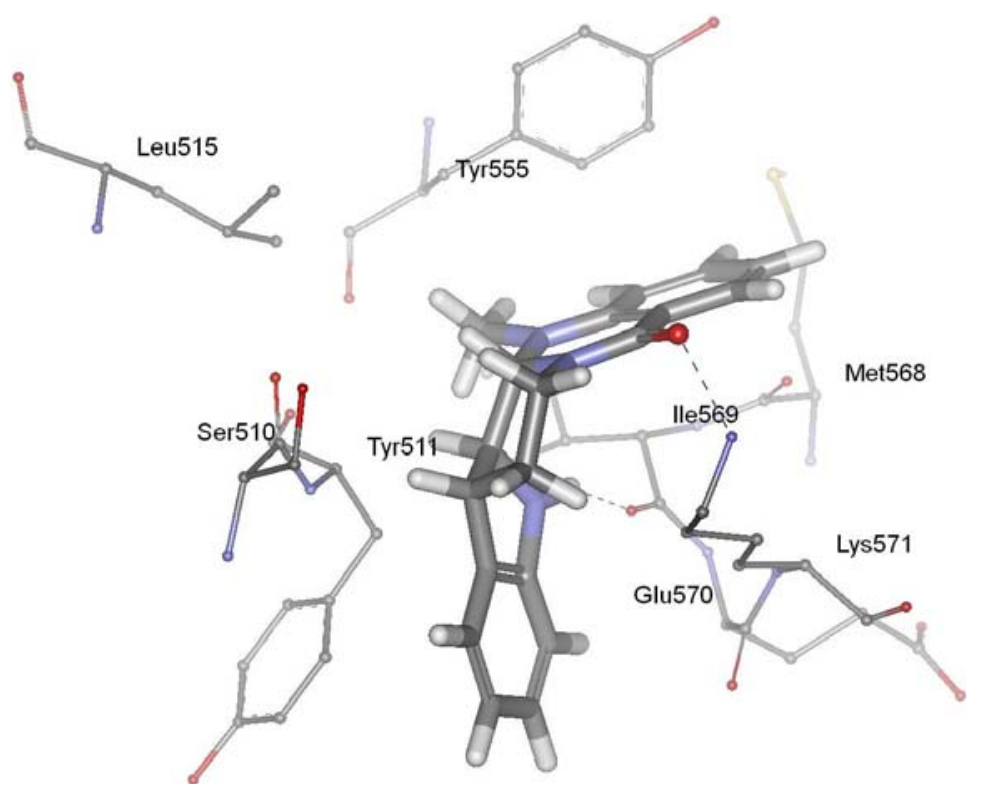

\subsection{DNA Topoisomerases}

DNA topoisomerases (I and II) are complex enzymes, which control the topological state of DNA throughout breaking and rejoining of DNA strands. Topoisomerases are involved in various DNA-related cellular processes, such as replication, transcription, recombination, chromatin condensation and daughter chromatides partitioning [108]. A lot of new data concerning basic features of the different types of topoisomerases was published. Since the structure and function were well characterized, topoisomerases have been shown to have a potential use for drug design. It is well known that the inhibitors of DNA topoisomerases showed pronounced antitumor activity [109-111]. Topoisomerases have been also shown to have a potential for delivering antibacterial compounds or drug candidates [112,113]. In addition, topoisomerases are involved in the cardiovascular and nervous system diseases [114,115]. Dong et al. gained a better understanding of the probable binding modes of evodiamine within the human topoisomerase I binding pocket. They also designed and synthesized a series of evodiamine analogues and investigated their structure-activity relationship [48]. Various groups were introduced to the indole nitrogen atom of evodiamine, and the substituted benzoyl groups were found to be favorable 
for the antitumor activity. The result of the biological activity test showed that the 4- $\mathrm{Cl}$ benzoyl derivative was the most active one with $\mathrm{IC}_{50}$ values in the range $0.049-2.6 \mu \mathrm{M}$ [48]. Figure 5 shows the possible binding mode of evodiamine and 3-(4-chlorobenzoyl) evodiamine in the active site of topoisomerase I-DNA complex.

Figure 5. Binding modes of evodiamine and 3-(4-chlorobenzoyl) evodiamine in the active site of topoisomerase I-DNA complex.

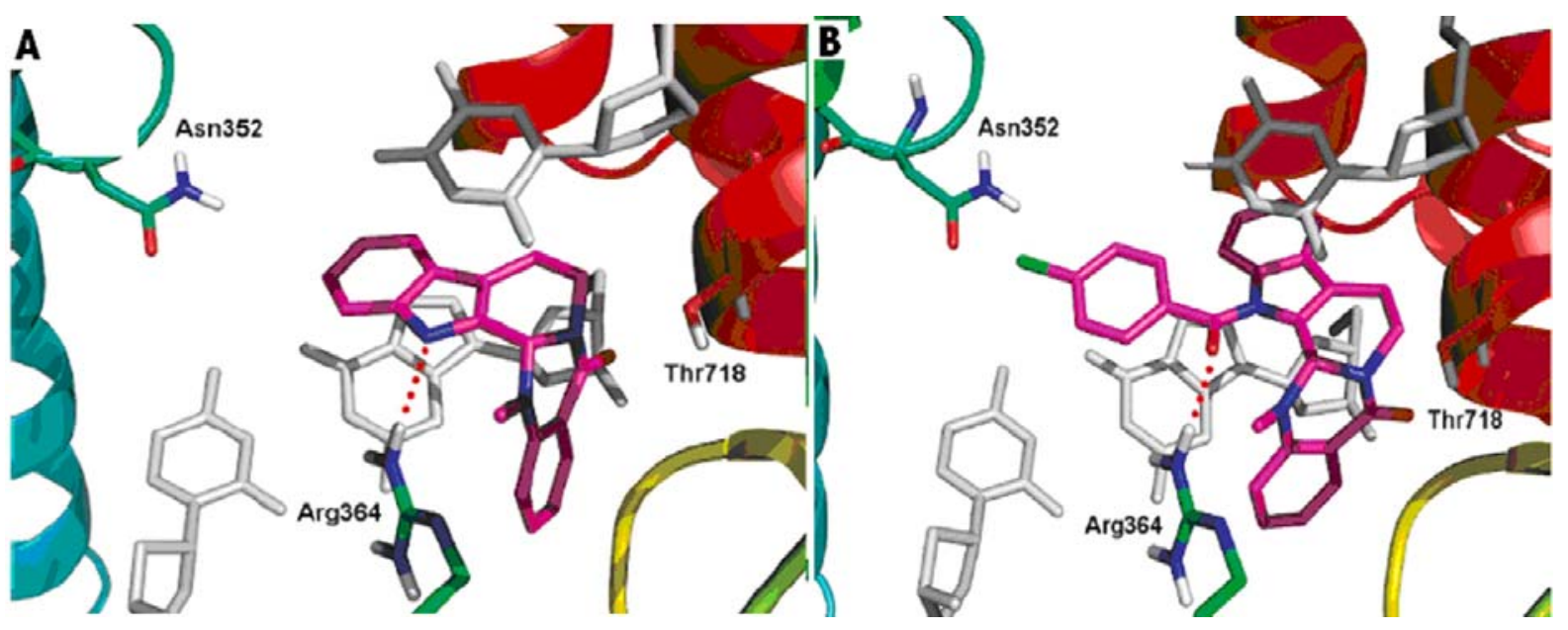

\subsection{Aryl Hydrocarbon Receptor}

The AhR is a member of the family of basic helix-loop-helix/Per-Arnt-Sim (bHLH/PAS) transcription factors [116]. Non-ligand bound AhR is retained in the cytoplasm as an inactive protein complex bound to several co-chaperones [117-119]. Upon ligand binding to AhR, the chaperones are released, AhR imported into the nucleus and dimerized with AhR nuclear translocator (ARNT), leading to changes in gene transcription [120-122]. AhR plays a functional role in physiology and toxicology, especially in cellular proliferation and differentiation, the adaptive response, the toxic response, secretion of hormones and immunomodulation [123-127]. Recently, scientists found that AhR activation seems to be also important for cancer and inflammation, supporting the possibility of targeting the AhR for therapy in inflammation and a number of cancers [128-130]. Recently, we investigated the interaction between evodiamine and AhR [131]. We observed that a series of hydrophobic residues of AhR are involved in complex formation: Phe285, Phe295, Gly304, Val307, Leu308, Ile325, Cys333, Met348, Val363, and Ser365 (Figure 6). However, there is any hydrogen bond between AhR and evodiamine. Competitive ligand binding assay showed that evodiamine inhibited the specific binding of $\left[{ }^{3} \mathrm{H}\right]-\mathrm{TCDD}$, the best-characterised AhR agonist, to the AhR with an $\mathrm{IC}_{50}$ value of $44.8 \pm 6.5 \mathrm{nM}$. The corresponding Ki value calculated for evodiamine was $28.4 \pm 4.9 \mathrm{nM}[131]$. AhR binding studies in vitro provided the evidence that evodiamine was able to bind to the AhR as ligand and exhibit antagonistic effects.

\section{Pharmacokinetics}

Evodiamine is sparingly soluble. Some efforts have been done to improve the bioavailability of evodiamine. Previous studies have revealed that solid dispersions of evodiamine in hard capsules have 
a greater absorption rate than enriched samples of evodiamine in physical mixture hard capsules [132]. Tan et al. designed a novel evodiamine-phospholipid complex, which has higher bioavailability than evodiamine [133]. The pharmacokinetics of evodiamine were investigated in rats $[134,135]$. Recent research indicated that the plasma concentration of evodiamine reached the maximum level within $1 \mathrm{~h}$ after oral administration, and 19\% of orally administered evodiamine was excreted in urine after $24 \mathrm{~h}$. The main pharmacokinetic parameters were also calculated after oral administration of $\mathrm{Wu}-\mathrm{Chu}-\mathrm{Yu}$ extracts with different purities to rats. Additionally, Lin et al. developed a sensitive and selective liquid chromatography-mass spectrometry method for the determination of evodiamine in rabbit plasma for pharmacokinetic study [136].

Figure 6. Docking orientation of evodiamine within the AhR-LBD binding pocket.

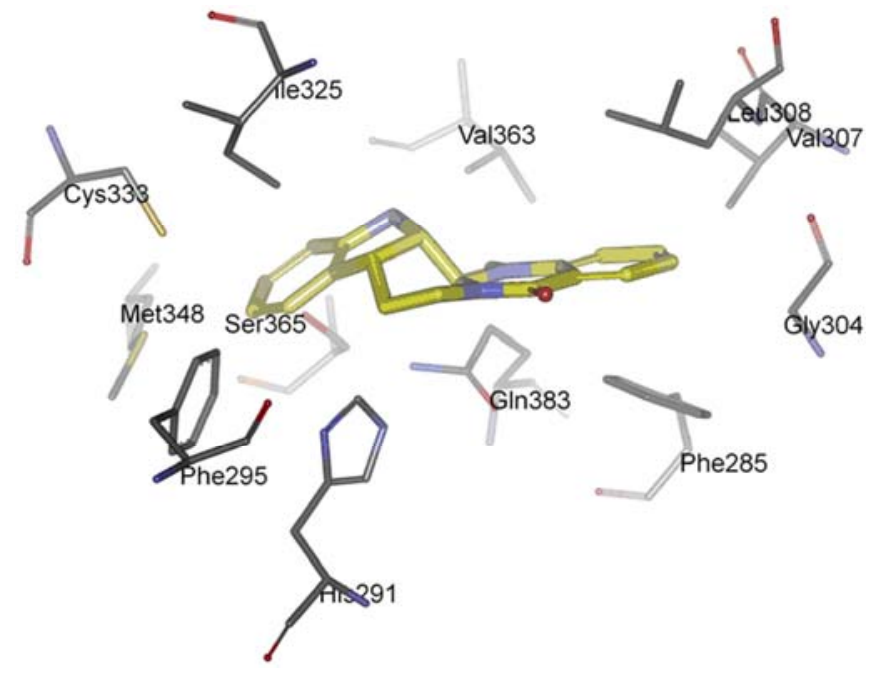

\section{Conclusions}

This review focuses on potent and diverse bioactivities of evodiamine reported in the recent years. The target-binding properties of evodiamine were also highlighted. Information provided in this manuscript can be useful not only to exploit their biological potential appropriately, but also to develop multi-target drugs of future for treatment of various diseases.

\section{Acknowledgments}

This work was supported by grants from the National Basic Research Program of China (No. 2012CB518102), the National Natural Science Foundation of China (No. 81260478, No. 81171801, No. 81201497), the Natural Science Foundation of Inner Mongolia Autonomous Region, China (No. 2011BS1201), the Chongqing Science and Technology Committee (No. CSTC2012jjA0171), the Project of State Key Laboratory of Trauma, Burns, and Combined Injury (No. SKLKF201205, No. SKLZZ201108), the Project of State Key Laboratory of Natural and Biomimetic Drugs (No. K20120205) and the Science and Technology Support Program of Baotou (2012S2006-04-09). 


\section{References}

1. Wang, Y.; Fan, X.; Qu, H.; Gao, X.; Cheng, Y. Strategies and techniques for multi-component drug design from medicinal herbs and traditional chinese medicine. Curr. Top Med. Chem. 2012, $12,1356-1362$.

2. Petrelli, A.; Giordano, S. From single- to multi-target drugs in cancer therapy: When aspecificity becomes an advantage. Curr. Med. Chem. 2008, 15, 422-432.

3. Tong, X.L.; Dong, L.; Chen, L.; Zhen, Z. Treatment of diabetes using traditional Chinese medicine: Past, present and future. Am. J. Chin. Med. 2012, 40, 877-886.

4. Wang, S.; Wu, X.; Tan, M.; Gong, J.; Tan, W.; Bian, B.; Chen, M.; Wang, Y. Fighting fire with fire: Poisonous Chinese herbal medicine for cancer therapy. J. Ethnopharmacol. 2012, 140, 33-45.

5. Chen, Y.F. Traditional Chinese herbal medicine and cerebral ischemia. Front Biosci. (Elite Ed.) 2012, 4, 809-817.

6. Liu, Z.L.; Liu, J.P.; Zhang, A.L.; Wu, Q.; Ruan, Y.; Lewith, G.; Visconte, D. Chinese herbal medicines for hypercholesterolemia. Cochrane Database Syst. Rev. 2011, 7, CD008305.

7. Yue, R.; Shan, L.; Yang, X.; Zhang, W. Approaches to target profiling of natural products. Curr. Med. Chem. 2012, 19, 3841-3855.

8. Zhao, J.; Yang, P.; Li, F.; Tao, L.; Ding, H.; Rui, Y.; Cao, Z.; Zhang, W. Therapeutic effects of astragaloside IV on myocardial injuries: Multi-target identification and network analysis. PLoS One 2012, 7, e44938.

9. Liao, J.F.; Chiou, W.F.; Shen, Y.C.; Wang, G.J.; Chen, C.F. Anti-inflammatory and anti-infectious effects of Evodia rutaecarpa (Wuzhuyu) and its major bioactive components. Chin. Med. 2011, 6, 6-13.

10. Moon, T.C.; Murakami, M.; Kudo, I.; Son, K.H.; Kim, H.P.; Kang, S.S.; Chang, H.W. A new class of COX-2 inhibitor, rutaecarpine from Evodia rutaecarpa. Inflamm. Res. 1999, 48, 621-625.

11. Choi, Y.H.; Shin, E.M.; Kim, Y.S.; Cai, X.F.; Lee, J.J.; Kim, H.P. Anti-inflammatory principles from the fruits of Evodia rutaecarpa and their cellular action mechanisms. Arch. Pharm. Res. 2006, 29, 293-297.

12. Kobayashi, Y.; Nakano, Y.; Kizaki, M.; Hoshikuma, K.; Yokoo, Y.; Kamiya, T. Capsaicin-like anti-obese activities of evodiamine from fruits of Evodia rutaecarpa, a vanilloid receptor agonist. Planta Med. 2001, 67, 628-633.

13. Fei, X.F.; Wang, B.X.; Li, T.J.; Tashiro, S.; Minami, M.; Xing, D.J.; Ikejima, T. Evodiamine, a constituent of Evodiae Fructus, induces anti-proliferating effects in tumor cells. Cancer Sci. 2003, 94, 92-98.

14. Kobayashi, Y. The nociceptive and anti-nociceptive effects of evodiamine from fruits of Evodia rutaecarpa in mice. Planta Med. 2003, 69, 425-428.

15. Nathan, C. Nitric oxide as a secretory product of mammalian cells. FASEB J. 1992, 6, 3051-3064.

16. Chiou, W.F.; Sung, Y.J.; Liao, J.F.; Shum, A.Y.; Chen, C.F. Inhibitory effect of dehydroevodiamine and evodiamine on nitric oxide production in cultured murine macrophages. J. Nat. Prod. 1997, $60,708-711$. 
17. Ko, H.C.; Wang, Y.H.; Liou, K.T.; Chen, C.M.; Chen, C.H.; Wang, W.Y.; Chang, S.; Hou, Y.C.; Chen, K.T.; Chen, C.F.; et al. Anti-inflammatory effects and mechanisms of the ethanol extract of Evodia rutaecarpa and its bioactive components on neutrophils and microglial cells. Eur. J. Pharmacol. 2007, 555, 211-217.

18. Liu, Y.N.; Pan, S.L.; Liao, C.H.; Huang, D.Y.; Guh, J.H.; Peng, C.Y.; Chang, Y.L.; Teng, C.M. Evodiamine represses hypoxia-induced inflammatory proteins expression and hypoxia-inducible factor 1alpha accumulation in RAW264.7. Shock 2009, 32, 263-269.

19. Lin, C.R.; Amaya, F.; Barrett, L.; Wang, H.; Takada, J.; Samad, T.A.; Woolf, C.J. Prostaglandin E2 receptor EP4 contributes to inflammatory pain hypersensitivity. J. Pharmacol. Exp. Ther. 2006, 319, 1096-1103.

20. Ogasawara, M.; Matsubara, T.; Suzuki, H. Inhibitory effects of evodiamine on in vitro invasion and experimental lung metastasis of murine colon cancer cells. Biol. Pharm. Bull. 2001, 24, 917-920.

21. Ogasawara, M.; Matsubara, T.; Takahashi, S.; Saiki, I.; Suzuki, H. Anti-invasive and metastatic activities of evodiamine. Biol. Pharm. Bull. 2002, 25, 1491-1493.

22. Wang, C.; Wang, M.W.; Tashiro, S.; Onodera, S.; Ikejima, T. Evodiamine induced human melanoma A375-S2 cell death partially through interleukin 1 mediated pathway. Biol. Pharm. Bull. 2005, 28, 984-989.

23. Lee, T.J.; Kim, E.J.; Kim, S.; Jung, E.M.; Park, J.W.; Jeong, S.H.; Park, S.E.; Yoo, Y.H.; Kwon, T.K. Caspase-dependent and caspase-independent apoptosis induced by evodiamine in human leukemic U937 cells. Mol. Cancer Ther. 2006, 5, 2398-2407.

24. Huang, Y.C.; Guh, J.H.; Teng, C.M. Induction of mitotic arrest and apoptosis by evodiamine in human leukemic T-lymphocytes. Life Sci. 2004, 75, 35-49.

25. Kan, S.F.; Yu, C.H.; Pu, H.F.; Hsu, J.M.; Chen, M.J.; Wang, P.S. Anti-proliferative effects of evodiamine on human prostate cancer cell lines DU145 and PC3. J. Cell. Biochem. 2007, 101, 44-56.

26. Huang, D.M.; Guh, J.H.; Huang, Y.T.; Chueh, S.C.; Chiang, P.C.; Teng, C.M. Induction of mitotic arrest and apoptosis in human prostate cancer PC-3 cells by evodiamine. J. Urol. 2005, 173, 256-261.

27. Kang, S.F.; Huang, W.J.; Lin, L.C.; Wang, P.S. Inhibitory effects of evodiamine on the growth of human prostate cancer cell line LNCaP. Int. J. Cancer 2004, 110, 641-651.

28. Liao, C.H.; Pan, S.L.; Guh, J.H.; Chang, Y.L.; Pai, H.C.; Lin, C.H.; Teng, C.M. Antitumor mechanism of evodiamine, a constituent from Chinese herb Evodiae fructus, in human multiple-drug resistant breast cancer NCI/ADR-RES cells in vitro and in vivo. Carcinogenesis 2005, 26, 968-975.

29. Jiang, J.; Hu, C. Evodiamine: A novel anti-cancer alkaloid from Evodia rutaecarpa. Molecules 2009, 14, 1852-1859.

30. Zhang. Y.; Wu, L.J.; Tashiro, S.; Onodera, S.; Ikejima, T. Intracellular regulation of evodiamine-induced A375-S2 cell death. Biol. Pharm. Bull. 2003, 26, 1543-1547.

31. Zhang. Y.; Wu, L.J.; Tashiro, S.; Onodera, S.; Ikejima, T. Evodiamine induces tumor cell death through two different pathways: Apoptosis and necrosis. Acta Pharmacol. Sin. 2004, 25, 83-89.

32. Zhang, Y.; Zhang, Q.H.; Wu, L.J.; Tashiro, S.; Onodera, S.; Ikejima, T. A typical apoptosis in L929 cells induced by evodiamine isolated from Evodia rutaecarpa. J. Asian Nat. Prod. Res. 2004, 6, 19-27. 
33. Takada, Y.; Kobayashi, Y.; Aggarwal, B.B. Evodiamine abolishes constitutive and inducible NF-kappaB activation by inhibiting IkappaBalpha kinase activation, thereby suppressing NF-kappaB-regulated antiapoptotic and metastatic gene expression, up-regulating apoptosis, and inhibiting invasion. J. Biol. Chem. 2005, 280, 17203-17212.

34. Chen, M.C.; Yu, C.H.; Wang, S.W.; Pu, H.F.; Kan, S.F.; Lin, L.C.; Chi, C.W.; Ho, L.L.; Lee, C.H.; Wang, P.S. Anti-proliferative effects of evodiamine on human thyroid cancer cell line ARO. J. Cell. Biochem. 2010, 110, 1495-1503.

35. Zhang, C.; Fan, X.; Xu, X.; Yang, X.; Wang, X.; Liang, H.P. Evodiamine induces caspase-dependent apoptosis and $\mathrm{S}$ phase arrest in human colon lovo cells. Anticancer Drugs 2010, 21, 766-776.

36. Yang, J.; Wu, L.J.; Tashino, S.; Onodera, S.; Ikejima, T. Critical roles of reactive oxygen species in mitochondrial permeability transition in mediating evodiamine-induced human melanoma A375-S2 cell apoptosis. Free Radic. Res. 2007, 41, 1099-1108.

37. Yang, J.; Wu, L.J.; Tashiro, S.; Onodera, S.; Ikejima, T. Nitric oxide activated by p38 and NF-kappaB facilitates apoptosis and cell cycle arrest under oxidative stress in evodiamine-treated human melanoma A375-S2 cells. Free Radic. Res. 2008, 42, 1-11.

38. Yang, J.; Wu, L.J.; Tashino, S.; Onodera, S.; Ikejima, T. Reactive oxygen species and nitric oxide regulate mitochondria-dependent apoptosis and autophagy in evodiamine-treated human cervix carcinoma HeLa cells. Free Radic. Res. 2008, 42, 492-504.

39. Yang, J.; Wu, L.J.; Tashino, S.; Onodera, S.; Ikejima, T. Protein tyrosine kinase pathway-derived ROS/NO productions contribute to G2/M cell cycle arrest in evodiamine-treated human cervix carcinoma Hela cells. Free Radic. Res. 2010, 44, 792-802.

40. Wang, C.; Li, S.; Wang, M.W. Evodiamine-induced human melanoma A375-S2 cell death was mediated by PI3K/Akt/caspase and Fas-L/NF-kappaB signaling pathways and augmented by ubiquitin-proteasome inhibition. Toxicol. In Vitro 2010, 24, 898-904.

41. Wei, W.T.; Chen, H.; Wang, Z.H.; Ni, Z.L.; Liu, H.B.; Tong, H.F.; Guo, H.C.; Liu, D.L.; Lin, S.Z. Enhanced antitumor efficacy of gemcitabine by evodiamine on pancreatic cancer via regulating PI3K/Akt pathway. Int. J. Biol. Sci. 2012, 8, 1-14.

42. Chao, D.C.; Lin, L.J.; Hsiang, C.Y.; Li, C.C.; Lo, H.Y.; Liang, J.A.; Kao, S.T.; Wu, S.L.; Ho, T.Y. Evodiamine inhibits 12-O-tetradecanoylphorbol-13-acetate-induced activator protein 1 transactivation and cell transformation in human hepatocytes. Phytother. Res. 2011, 25, 1018-1023.

43. Shyu, K.G.; Lin, S.; Lee, C.C.; Chen, E.; Lin, L.C.; Wang, B.W.; Tsai, S.C. Evodiamine inhibits in vitro angiogenesis: Implication for antitumorgenicity. Life Sci. 2006, 78, 2234-2243.

44. Ogasawara, M.; Suzuki, H. Inhibition by evodiamine of hepatocyte growth factor-induced invasion and migration of tumor cells. Biol. Pharm. Bull. 2004, 27, 578-582.

45. Pan, X.; Hartley, J.M.; Hartley, J.A.; White, K.N.; Wang, Z.; Bligh, S.W. Evodiamine, a dual catalytic inhibitor of type I and II topoisomerases, exhibits enhanced inhibition against camptothecin resistant cells. Phytomedicine 2012, 19, 618-624.

46. Chan, A.L.; Chang, W.S.; Chen, L.M.; Lee, C.M.; Chen, C.E.; Lin, C.M.; Hwang, J.L. Evodiamine stabilizes topoisomerase I-DNA cleavable complex to inhibit topoisomerase I activity. Molecules 2009, 14, 1342-1352.

47. Sheng, C.; Miao, Z.; Zhang, W. New strategies in the discovery of novel non-camptothecin topoisomerase I inhibitors. Curr. Med. Chem. 2011, 18, 4389-4409. 
48. Dong, G.; Sheng, C.; Wang, S.; Miao, Z.; Yao, J.; Zhang, W. Selection of evodiamine as a novel topoisomerase I inhibitor by structure-based virtual screening and hit optimization of evodiamine derivatives as antitumor agents. J. Med. Chem. 2010, 53, 7521-7531.

49. Hu, Y.; Fahmy, H.; Zjawiony, J.K.; Davies, G.E. Inhibitory effect and transcriptional impact of berberine and evodiamine on human white preadipocyte differentiation. Fitoterapia 2010, 81, 259-268.

50. Shi, J.; Yan, J.; Lei, Q.; Zhao, J.; Chen, K.; Yang, D.; Zhao, X.; Zhang, Y. Intragastric administration of evodiamine suppresses NPY and AgRP gene expression in the hypothalamus and decreases food intake in rats. Brain Res. 2009, 1247, 71-78.

51. Wang, T.; Wang, Y.; Yamashita, H. Evodiamine inhibits adipogenesis via the EGFR-PKCalpha-ERK signaling pathway. FEBS Lett. 2009, 583, 3655-3659.

52. Wang, T.; Wang, Y.; Kontani, Y.; Kobayashi, Y.; Sato, Y.; Mori, N.; Yamashita, H. Evodiamine improves diet-induced obesity in a uncoupling protein-1-independent manner: Involvement of antiadipogenic mechanism and extracellularly regulated kinase/mitogen-activated protein kinase signaling. Endocrinology 2008, 149, 358-366.

53. Wu, C.L.; Hung, C.R.; Chang, F.Y.; Lin, L.C.; Pau, K.Y.; Wang, P.S. Effects of evodiamine on gastrointestinal motility in male rats. Eur. J. Pharmacol. 2002, 457, 169-176.

54. Vincent, R.P.; Ashrafian, H.; le Roux, C.W. Mechanisms of disease: The role of gastrointestinal hormones in appetite and obesity. Nat. Clin. Pract. Gastroenterol. Hepatol. 2008, 5, 268-277.

55. Yang, H.Y.; Li, S.Y.; Chen, C.F. Hypotensive effects of dehydroevodiamine, a quinazolinocarboline alkaloid isolated from Evodia rutaecarpa. Asia Pac. J. Pharmacol. 1988, 3, 191-195.

56. Yang, M.C.; Wu, S.L.; Kao, J.S.; Chen, C.F. The hypotensive and negative chronotropic effect of dehydroevodiamine. Eur. J. Pharmacol. 1990, 182, 537-542.

57. Chiou, W.F.; Chou, C.J.; Shum, A.Y.C.; Chen, C.F. The vasorelaxant effect of evodiamine in rat isolated mesenteric arteries: Mode of action. Eur. J. Pharmacol. 1992, 215, 277-283.

58. Chiou, W.F.; Liao, J.F.; Chen, C.F. Comparative study on vasodilatory effects of three quinazoline alkaloids isolated from Evodia rutaecarpa. J. Nat. Prod. 1996, 59, 374-378.

59. Chen, C.F.; Chen, S.M.; Lin, M.T.; Chow, S.Y. In vivo and in vitro studies on the mechanism of cardiovascular effects of Wu-Chu-Yu (Evodiae fructus). Am. J. Chin. Med. 1981, 9, 39-47.

60. Hung, P.H.; Lin, L.C.; Wang, G.J.; Chen, C.F.; Wang, P.S. Inhibitory effect of evodiamine on aldosterone release by Zona glomerulosa cells in male rats. Chin. J. Physiol. 2001, 44, 53-57.

61. Kobayashi, Y.; Hoshikuma, K.; Nakano, Y.; Yokoo, Y.; Kamiya, T. The positive inotropic and chronotropic effects of evodiamine and rutaecarpine, indoloquinazoline alkaloids isolated from the fruits of Evodia rutaecarpa, on the guinea-pig isolated right atria: Possible involvement of vanilloid receptors. Planta Med. 2001, 67, 244-248.

62. Rang, W.Q.; Du, Y.H.; Hu, C.P.; Ye, F.; Tan, G.S.; Deng, H.W.; Li, Y.J. Protective effects of calcitonin gene-related peptide-mediated evodiamine on guinea-pig cardiac anaphylaxis. Naunyn Schmiedebergs Arch. Pharmacol. 2003, 367, 306-311.

63. Yi, H.H.; Rang, W.Q.; Deng, P.Y.; Hu, C.P.; Liu, G.Z.; Tan, G.S.; Xu, K.P.; Li, Y.J. Protective effects of rutaecarpine in cardiac anaphylactic injury is mediated by CGRP. Planta Med. 2004, $70,1135-1139$. 
64. Yu, J.; Tan, G.S.; Deng, P.Y.; Xu, K.P.; Hu, C.P.; Li, Y.J. Involvement of CGRP in the inhibitory effect of rutaecarpine on vasoconstriction induced by anaphylaxis in guinea pig. Regul. Pept. 2005, 125, 93-97.

65. Rang, W.Q.; Du, Y.H.; Hu, C.P.; Ye, F.; Xu, K.P.; Peng, J.; Deng, H.W.; Li, Y.J. Protective effects of evodiamine on myocardial ischemia-reperfusion injury in rats. Planta Med. 2004, 70, $1140-1143$.

66. Hu, C.P.; Xiao, L.; Deng, H.W.; Li, Y.J. The cardioprotection of rutaecarpine is mediated by endogenous calcitonin release-gene peptide through activation of vanilloid receptors in guinea-pig hearts. Planta Med. 2002, 68, 705-709.

67. Heo, S.K.; Yun, H.J.; Yi, H.S.; Noh, E.K.; Park, S.D. Evodiamine and rutaecarpine inhibit migration by LIGHT via suppression of NADPH oxidase activation. J. Cell. Biochem. 2009, 107, 123-133.

68. Mattson, M.P. Pathways towards and away from Alzheimer's disease. Nature 2004, 430, 631-639.

69. Hardy, J.; Selkoe, D.J. The amyloid hypothesis of Alzheimer's disease: Progress and problems on the road to therapeutics. Science 2002, 297, 353-356.

70. Kim, H.K.; Kim, M.; Kim, S.; Kim, M.; Chung, J.H. Effects of green tea polyphenol on cognitive and acetylcholinesterase activities. Biosci. Biotechnol. Biochem. 2004, 68, 1977-1979.

71. Lu, J.H.; Guo, J.; Yang, W.H. Effects of green tea polyphenol on the behaviour of Alzheimer's disease like mice induced by D-galactose and Abeta $25-35$. Zhong Yao Cai 2006, 29, 352-354.

72. Marambaud, P.; Zhao, H.; Davies, P. Resveratrol promotes clearance of Alzheimer's disease amyloid-beta peptides. J. Biol. Chem. 2005, 280, 37377-37382.

73. Karuppagounder, S.S.; Pinto, J.T.; Xu, H.; Chen, H.L.; Beal, M.F.; Gibson, G.E. Dietary supplementation with resveratrol reduces plaque pathology in a transgenic model of Alzheimer's disease. Neurochem. Int. 2009, 54, 111-118.

74. Sehirli, O.; Tozan, A.; Omurtag, G.Z.; Cetinel, S.; Contuk, G.; Gedik, N.; Sener, G. Protective effect of resveratrol against naphthalene-induced oxidative stress in mice. Ecotoxicol. Environ. Saf. 2008, 71, 301-308.

75. Haji, A.; Momose, Y.; Takeda, R.; Nakanishi, S.; Hiriachi, T.; Arisawa, M. Increased feline cerebral blood flow induced by dehydroevodiamine hydrochloride from Evodia rutaecarpa. J. Nat. Prod. 1994, 57, 387-389.

76. Yuan, S.M.; Gao, K.; Wang, D.M.; Quan, X.Z.; Liu, J.N.; Ma, C.M.; Qin, C.; Zhang, L.F. Evodiamine improves congnitive abilities in SAMP8 and APP(swe)/PS1( $\triangle \mathrm{E} 9)$ transgenic mouse models of Alzheimer's disease. Acta Pharmacol. Sin. 2011, 32, 295-302.

77. Park, C.H.; Kim, S.H.; Choi, W.; Lee, Y.J.; Kim, J.S.; Kang, S.S.; Suh, Y.H. Novel anticholinesterase and antiamnesic activities of dehydroevodiamine, a constituent of Evodia rutaecarpa. Planta Med. 1996, 62, 405-409.

78. Perrett, S.; Whitfield, P.J. Atanine (3-dimethylallyl-4-methoxy-2-quinolone), an alkaloid with anthelmintic activity from the Chinese medicinal plant, Evodia rutaecarpa. Planta Med. 1995, 61, 276-278.

79. Rho, T.C.; Bae, E.A.; Kim, D.H.; Oh, W.K.; Kim, B.Y.; Ahn, J.S.; Lee, H.S. Anti-Helicobacter pylori activity of quinolone alkaloids from Evodiae Fructus. Biol. Pharm. Bull. 1999, 22, 1141-1143. 
80. Hamasaki, N.; Ishii, E.; Tominaga, K.; Tezuka, Y.; Nagaoka, T.; Kadota, S.; Kuroki, T.; Yano, I. Highly selective antibacterial activity of novel alkyl quinolone alkaloids from a Chinese herbal medicine, Gosyuyu (Wu-Chu-Yu), against Helicobacter pylori in vitro. Microbiol. Immunol. 2000, 44, 9-15.

81. Tominaga, K.; Higuchi, K.; Hamasaki, N.; Hamaguchi, M.; Takashima, T.; Tanigawa, T.; Watanabe, T.; Fujiwara, Y.; Tezuke, Y.; Nagaoka, T.; et al. In vivo action of novel alkyl methyl quinolone alkaloids against Helicobacter pylori. J. Antimicrob. Chemother. 2002, 50, 547-552.

82. Tominaga, K.; Higuchi, K.; Hamasaki, N.; Tanigawa, T.; Sasaki, E.; Watanabe, T.; Fujiwara, Y.; Oshitani, N.; Arakawa, T.; Ishii, E.; et al. Antibacterial activity of a Chinese herb medicine, Gosyuy (Wu-Chu-Yu), against Helicobacter pylori. Nippon. Rinsho. 2005, 63, 592-599.

83. Chiou, W.F.; Ko, H.C.; Wei, B.L. Evodia rutaecarpa and three major alkaloids abrogate influenza A virus (H1N1)-induced chemokines production and cell migration. Evid. Based Complement. Alternat. Med. 2011, 2011, 750513.

84. Caterina, M.; Julius, D. The vanilloid receptor: A molecular gateway to the pain pathway. Annu. Rev. Neurosci. 2001, 24, 487-517.

85. Pearce, L.V.; Petukhov, P.A.; Szabo, T.; Kedei, N.; Bizik, F.; Kozikowski, A.P.; Blumberg, P.M. Evodiamine functions as an agonist for the vanilloid receptor TRPV1. Org. Biomol. Chem. 2004, 2, 2281-2286.

86. Peng, L.; Li, Y.J. The vanilloid receptor TRPV-1: Role in cardiovascular and gastrointestinal protection. Eur. J. Pharmacol. 2010, 627, 1-7.

87. Matsuda, H.; Wu, T.X.; Tanaka, T.; Linuma, M.; Kubo, M. Antinociceptive activities of 70\% methanol extract of Evodiae Fructus (fruit of Evodia rutaecarpavar. bodinieri) and its alkaloidal components. Biol. Pharm. Bull. 1997, 20, 243-248.

88. Kano, Y.; Zong, Q.N.; Komatsu, K. Pharmacological properties of galenical preparation, XIV. Body temperature retaining effect of the Chinese traditional medicine, "goshuyu-to" and component crude drugs. Chem. Pharm. Bull. (Tokyo) 1991, 39, 690-692.

89. Tsai, T.H.; Lee, T.F.; Chen, C.F.; Wang, L.C. Thermoregulatory effects of alkaloids isolated from Wu-Chu-Yu in afebrile and febrile rats. Pharmacol. Biochem. Behav. 1995, 50, 293-298.

90. Lin, H.; Tsai, S.C.; Chen, J.J.; Chiao, Y.C.; Wang, S.W.; Wang, G.J.; Chen, C.F.; Wang, P.S. Effects of evodiamine on the secretion of testosterone in rat testicular interstitial cells. Metabolism 1999, 48, 1532-1535.

91. Yoshizumi, M.; Houchi, H.; Ishimura, Y.; Hirose, M.; Kitagawa, T.; Tsuchiya, K.; Minakuchi, K.; Tamaki, T. Effect of evodiamine on catecholamine secretion from bovine adrenal medulla. J. Med. Invest. 1997, 44, 79-82.

92. Yu, P.L.; Chao, H.L.; Wang, S.W.; Wang, P.S. Effects of evodiamine and rutaecarpine on the secretion of corticosterone by zona fasciculata-reticularis cells in male rats. J. Cell. Biochem. 2009, 108, 469-475.

93. Yamahara, J.; Yamada, T.; Kitani, T.; Naitoh, Y.; Fujimura, H. Antianoxic action of evodiamine, an alkaloid in Evodia rutaecarpa fruit. J. Ethnopharmacol. 1989, 27, 185-192.

94. Shin, Y.W.; Bae, E.A.; Cai, X.F.; Lee, J.J.; Kim, D.H. In vitro and in vivo antiallergic effects on the fruits of Evodia rutaecarpa and its constituents. Biol. Pharm. Bull. 2007, 30, 197-199. 
95. Hu, Y.; Ehli, E.A.; Hudziak, J.J.; Davies, G.E. Berberine and evodiamine influence serotonin transporter (5-HTT) expression via the 5-HTT-linked polymorphic region. Pharmacogenomics $J$. 2012, 12, 372-378.

96. Kobayashi, Y.; Nakano, Y.; Hoshikuma, K.; Yokoo, Y.; Kamiya, T. The bronchoconstrictive action of evodiamine, an indoloquinazoline alkaloid isolated from the fruits of Evodia rutaecarpa, on guinea-pig isolated bronchus: Possible involvement on vanilloid receptors. Planta Med. 2000, $66,526-530$.

97. Guerrant, W.; Patil, V.; Canzoneri, J.C.; Oyelere, A.K. Dual targeting of histone deacetylase and topoisomerase II with novel bifunctional inhibitors. J. Med. Chem. 2012, 55, 1465-1477.

98. Wei, J.; Ching, L.C.; Zhao, J.F.; Shyue, S.K.; Lee, H.F.; Kou, Y.R.; Lee, T.S. Essential role of transient receptor potential vanilloid type 1 in evodiamine-mediated protection against atherosclerosis. Acta Physiol. (Oxf.) 2013, 207, 299-307.

99. Devesa, I.; Planells-Cases, R.; Fernández-Ballester, G.; González-Ros, J.M.; Ferrer-Montiel, A.; Fernández-Carvajal, A. Role of the transient receptor potential vanilloid 1 in inflammation and sepsis. J. Inflamm. Res. 2011, 4, 67-81.

100. Wanner, S.P.; Garami, A.; Pakai, E.; Oliveira, D.L.; Gavva, N.R.; Coimbra, C.C.; Romanovsky, A.A. Aging reverses the role of the transient receptor potential vanilloid-1 channel in systemic inflammation from anti-inflammatory to proinflammatory. Cell Cycle 2012, 11, 343-349.

101. Palazzo, E.; Luongo, L.; de Novellis, V.; Rossi, F.; Marabese, I.; Maione, S. Transient receptor potential vanilloid type 1 and pain development. Curr. Opin. Pharmacol. 2012, 12, 9-17.

102. Birerdinc, A.; Jarrar, M.; Stotish, T.; Randhawa, M.; Baranova, A. Manipulating molecular switches in brown adipocytes and their precursors: A therapeutic potential. Prog. Lipid Res. 2012, 52, 51-61.

103. Luo, Z.; Ma, L.; Zhao, Z.; He, H.; Yang, D.; Feng, X.; Ma, S.; Chen, X.; Zhu, T.; Cao, T.; et al. TRPV1 activation improves exercise endurance and energy metabolism through PGC-1 $\alpha$ upregulation in mice. Cell Res. 2012, 22, 551-564.

104. Mori, F.; Ribolsi, M.; Kusayanagi, H.; Monteleone, F.; Mantovani, V.; Buttari, F.; Marasco, E.; Bernardi, G.; Maccarrone, M.; Centonze, D. TRPV1 channels regulate cortical excitability in humans. J. Neurosci. 2012, 32, 873-879.

105. Lim, K.M.; Park, Y.H. Development of PAC-14028, a novel transient receptor potential vanilloid type 1 (TRPV1) channel antagonist as a new drug for refractory skin diseases. Arch. Pharm. Res. 2012, 35, 393-396.

106. Santoni, G.; Caprodossi, S.; Farfariello, V.; Liberati, S.; Gismondi, A.; Amantini, C. Antioncogenic effects of transient receptor potential vanilloid 1 in the progression of transitional urothelial cancer of human bladder. ISRN Urol. 2012, 2012, 458238.

107. Wang, Z.; Sun, L.; Yu, H.; Zhang, Y.; Gong, W.; Jin, H.; Zhang, L.; Liang, H. Binding mode prediction of evodiamine within vanilloid receptor TRPV1. Int. J. Mol. Sci. 2012, 13, 8958-8969.

108. Giliarov, D.A.; Shkundina, I.S. DNA-topoisomerases and their functions in cell. Mol. Biol. (Mosk.) 2012, 46, 52-63.

109. Thai, K.M.; Bui, Q.H.; Tran, T.D.; Huynh, T.N. QSAR modeling on benzo[c]phenanthridine analogues as topoisomerase I inhibitors and anti-cancer agents. Molecules 2012, 17, 5690-5712. 
110. Karki, R.; Thapa, P.; Yoo, H.Y.; Kadayat, T.M.; Park, P.H.; Na, Y.; Lee, E.; Jeon, K.H.; Cho, W.J.; Choi, H.; et al. Dihydroxylated 2,4,6-triphenyl pyridines: Synthesis, topoisomerase I and II inhibitory activity, cytotoxicity, and structure-activity relationship study. Eur. J. Med. Chem. 2012, 49, 219-228.

111. Lee, C.H.; Hsieh, M.Y.; Hsin, L.W.; Chen, H.C.; Lo, S.C.; Fan, J.R.; Chen, W.R.; Chen, H.W.; Chan, N.L.; Li, T.K. Anthracenedione-methionine conjugates are novel topoisomerase II-targeting anticancer agents with favorable drug resistance profiles. Biochem. Pharmacol. 2012, 83, 1208-1216.

112. Sanyal, G.; Doig, P. Bacterial DNA replication enzymes as targets for antibacterial drug discovery. Expert Opin. Drug Discov. 2012, 7, 327-339.

113. Hiltensperger, G.; Jones, N.G.; Niedermeier, S.; Stich, A.; Kaiser, M.; Jung, J.; Puhl, S.; Damme, A.; Braunschweig, H.; Meinel, L.; et al. Synthesis and structure-activity relationships of new quinolone-type molecules against Trypanosoma brucei. J. Med. Chem. 2012, 55, 2538-2548.

114. Martin, E.; Thougaard, A.V.; Grauslund, M.; Jensen, P.B.; Bjorkling, F.; Hasinoff, B.B.; Tjørnelund, J.; Sehested, M.; Jensen, L.H. Evaluation of the topoisomerase II-inactive bisdioxopiperazine ICRF-161 as a protectant against doxorubicin-induced cardiomyopathy. Toxicology 2009, 255, $72-79$.

115. Gupta, K.P.; Swain, U.; Rao, K.S.; Kondapi, A.K. Topoisomerase II $\beta$ regulates base excision repair capacity of neurons. Mech. Ageing Dev. 2012, 133, 203-213.

116. Burbach, K.M.; Poland, A.; Bradfield, C.A. Cloning of the Ah-receptor cDNA reveals a distinctive ligand-activated transcription factor. Proc. Natl. Acad. Sci. USA 1992, 89, 8185-8189.

117. Perdew, G.H. Association of the Ah receptor with the $90-\mathrm{kDa}$ heat shock protein. J. Biol. Chem. 1988, 263, 13802-13805.

118. Kazlauskas, A.; Poellinger, L.; Pongratz, I. Evidence that the co-chaperone p23 regulates ligand responsiveness of the dioxin (Aryl hydrocarbon) receptor. J. Biol. Chem. 1999, 274, 13519-13524.

119. Meyer, B.K.; Pray-Grant, M.G.; Vanden Heuvel, J.P.; Perdew, G.H. Hepatitis B virus $\mathrm{X}$-associated protein 2 is a subunit of the unliganded aryl hydrocarbon receptor core complex and exhibits transcriptional enhancer activity. Mol. Cell. Biol. 1998, 18, 978-988.

120. Pollenz, R.S.; Barbour, E.R. Analysis of the complex relationship between nuclear export and aryl hydrocarbon receptor-mediated gene regulation. Mol. Cell. Biol. 2000, 20, 6095-6104.

121. Hoffman, E.C.; Reyes, H.; Chu, F.F.; Sander, F.; Conley, L.H.; Brooks, B.A.; Hankinson, O. Cloning of a factor required for activity of the Ah (dioxin) receptor. Science 1991, 252, 954-958.

122. Reyes, H.; Reisz-Porszasz, S.; Hankinson, O. Identification of the Ah receptor nuclear translocator protein (Arnt) as a component of the DNA binding form of the Ah receptor. Science 1992, 256, 1193-1195.

123. Tijet, N.; Boutros, P.C.; Moffat, I.D.; Okey, A.B.; Tuomisto, J.; Pohjanvirta, R. Aryl hydrocarbon receptor regulates distinct dioxin-dependent and dioxin-independent gene batteries. Mol. Pharmacol. 2006, 69, 140-153.

124. Israel, D.I.; Whitlock, J.P. Induction of mRNA specific for cytochrome P1-450 in wild type and variant mouse hepatoma cells. J. Biol. Chem. 1983, 258, 10390-10394. 
125. Harrigan, J.A.; Vezina, C.M.; McGarrigle, B.P.; Ersing, N.; Box, H.C.; Maccubbin, A.E.; Olson, J.R. DNA adduct formation in precision-cut rat liver and lung slices exposed to benzo[a]pyrene. Toxicol. Sci. 2004, 77, 307-314.

126. Mezrich, J.D.; Nguyen, L.P.; Kennedy, G.; Nukaya, M.; Fechner, J.H.; Zhang, X.; Xing, Y.; Bradfield, C.A. SU5416, a VEGF receptor inhibitor and ligand of the AhR, represents a new alternative for immunomodulation. PLoS One 2012, 7, e44547.

127. Lee, C.; Riddick, D.S. Aryl hydrocarbon receptor-dependence of dioxin's effects on constitutive mouse hepatic cytochromes $\mathrm{P} 450$ and growth hormone signaling components. Can. J. Physiol. Pharmacol. 2012, 90, 1354-1363.

128. O’Donnell, E.F.; Kopparapu, P.R.; Koch, D.C.; Jang, H.S.; Phillips, J.L.; Tanguay, R.L.; Kerkvliet, N.I.; Kolluri, S.K. The aryl hydrocarbon receptor mediates leflunomide-induced growth inhibition of melanoma cells. PLoS One 2012, 7, e40926.

129. Platten, M.; Litzenburger, U.; Wick, W. The aryl hydrocarbon receptor in tumor immunity. Oncoimmunology 2012, 1, 396-397.

130. Monteleone, I.; Rizzo, A.; Sarra, M.; Sica, G.; Sileri, P.; Biancone, L.; MacDonald, T.T.; Pallone, F.; Monteleone, G. Aryl hydrocarbon receptor-induced signals up-regulate IL-22 production and inhibit inflammation in the gastrointestinal tract. Gastroenterology 2011, 141, 237-248.

131. Yu, H.; Tu,Y.; Zhang, C.; Fan, X.; Wang, X.; Wang, Z.; Liang, H. Evodiamine as a novel antagonist of aryl hydrocarbon receptor. Biochem. Biophys. Res. Commun. 2010, 402, 94-98.

132. Xu, H.; Zhang, T.; Yang, H.; Xiao, X.; Bian, Y.; Si, D.; Liu, C. Preparation of evodiamine solid dispersions and its pharmacokinetics. Indian J. Pharm. Sci. 2011, 73, 276-281.

133. Tan, Q.; Liu, S.; Chen, X.; Wu, M.; Wang, H.; Yin, H.; He, D.; Xiong, H.; Zhang, J. Design and evaluation of a novel evodiamine-phospholipid complex for improved oral bioavailability. AAPS PharmSciTech. 2012, 13, 534-547.

134. Komatsu, K.; Wakame, K.; Kano, Y. Pharmacological properties of galenical preparation. XVI. Pharmacokinetics of evodiamine and the metabolite in rats. Biol. Pharm. Bull. 1993, 16, 935-938.

135. Jeng, K.F.; Lin, Y.H.; Lin, L.C.; Chou, C.J.; Tsai, T.H.; Chen, C.F. High-performance liquid chromatographic determination of evodiamine in rat plasma: Application to pharmacokinetic studies. J. Chromatogr. B Biomed. Appl. 1995, 668, 343-345.

136. Lin, C.; Pan, X.; Li, W.; Ma, J.; Pan, J.; Cai, J.; Wang, X.; Lin, G. Simultaneous determination of evodiamine and rutecarpine in rabbit plasma by LC-ESI-MS and its application to pharmacokinetics. Pharmazie 2011, 66, 920-923.

(C) 2013 by the authors; licensee MDPI, Basel, Switzerland. This article is an open access article distributed under the terms and conditions of the Creative Commons Attribution license (http://creativecommons.org/licenses/by/3.0/). 